\section{Antagonistic regulation of Yan nuclear export by Mae and Crm1 may increase the stringency of the Ras response}

\author{
Haiyun Song, ${ }^{1,4}$ Minghua Nie, ${ }^{1,2}$ Feng Qiao, ${ }^{1,2,3}$ \\ James U. Bowie, 1,2,3 and Albert J. Courey 1,2,5 \\ ${ }^{1}$ Department of Chemistry and Biochemistry, ${ }^{2}$ Molecular \\ Biology Institute, ${ }^{3}$ UCLA-DOE Institute of Genomics and \\ Proteomics, University of California, Los Angeles, \\ Los Angeles, California 90095, USA
}

Phosphorylation of Yan, a major target of Ras signaling, leads to Crm1-dependent Yan nuclear export, a response that is regulated by Yan polymerization. Yan SAM (sterile $\alpha$ motif) domain mutations preventing polymerization result in Ras-independent, but Crm1-dependent Yan nuclear export, suggesting that polymerization prevents Yan export. Mae, which depolymerizes Yan, competes with Crm1 for binding to Yan. Phosphorylation of Yan favors Crm1 in this competition and counteracts inhibition of nuclear export by Mae. These findings suggest that, prior to Ras activation, the Mae/Yan interaction blocks premature nuclear export of Yan monomers. After activation, transcriptional up-regulation of Mae apparently leads to complete depolymerization and export of Yan.

Received April 26, 2005; revised version accepted June 3, 2005.

Receptor tyrosine kinases (RTKs) such as the epidermal growth factor receptor (EGFR) and Sevenless receptor allow cells to adjust patterns and levels of gene expression in response to extracellular signals (Wassarman et al. 1995; Freeman 1996; Tan and Kim 1999; Shvartsman et al. 2002; Shilo 2003). The extracellular ligands that communicate these signals are sometimes distributed in concentration gradients that must be interpreted to give sharp thresholds of transcription factor activity. One of the best-characterized RTK-responsive transcription factors is the Yan transcriptional repressor (Lai and Rubin 1992; O'Neill et al. 1994). A number of recent studies suggest that interactions between Yan, the nuclear exportin Crm1, and the transcriptional coregulator Mae play essential roles in establishing the required threshold transcriptional responses (Baker et al. 2001; Tootle et al. 2003; Qiao et al. 2004).

The Drosophila Yan protein is an ETS family transcription factor that represses its target genes in the ab-

[Keywords: Yan; Mae; Crm1; SAM domain; Ras; nuclear export] ${ }^{4}$ Present address: Institute of Molecular Biology, University of Zurich, Winterthurerstrasse 190, CH-8057 Zurich, Switzerland.

${ }^{5}$ Corresponding author.

E-MAIL courey@chem.ucla.edu; FAX (310) 206-4038.

Article published online ahead of print. Article and publication date are

at http://www.genesdev.org/cgi/doi/10.1101/gad.1327405. sence of RTK signaling (Lai and Rubin 1992; O'Neill et al. 1994). Regulation of Yan nuclear export is a critical feature of RTK-induced cell differentiation. In unstimulated cells, Yan remains in the nucleus and antagonizes activation by Pointed (Pnt), another ETS family transcriptional regulator. Stimulation of the RTK pathway results in the activation of the Ras proto-oncoprotein, which in turn leads to the phosphorylation of Yan by the Rolled MAP kinase at Ser127. This phosphorylation triggers the Crm1-dependent nuclear export of Yan, thus allowing the activation of cell differentiation genes by Pnt (Klambt 1993; O'Neill et al. 1994; Rebay and Rubin 1995; Gabay et al. 1996; Tootle et al. 2003).

While it is clear that Yan phosphorylation enhances Yan nuclear export, the recognition of Yan by the nuclear export machinery is likely subject to multiple layers of regulation. This is suggested, for example, by the observation that mutations in the Yan DNA-binding domain result in Crm1-dependent nuclear export of Yan in the absence of RTK signaling (Tootle et al. 2003). A potential explanation for this unexpected finding is provided by studies of Yan polymerization. Yan contains an $\mathrm{N}$-terminal sterile $\alpha$ motif (SAM) domain that mediates the formation of a head-to-tail helical polymer; formation of this polymer appears to be required for transcriptional repression (Qiao et al. 2004). The N-terminal region of Yan, which includes the SAM domain, is required for the recognition of Yan by Crm1 (Tootle et al. 2003), strongly suggesting that Yan polymerization and Yan: Crml binding may be mutually exclusive. The association of Yan subunits in the polymer is of modest affinity $\left(\mathrm{K}_{\mathrm{d}} \approx 11 \mu \mathrm{M}\right)$, and it is possible that the sequence-specific or nonspecific binding of adjacent Yan monomers in the polymer to DNA stabilizes the Yan polymer, thereby masking the Crm1 interaction surface. In the absence of Yan polymerization, the Yan:Crm1 interaction may occur even in the absence of Yan phosphorylation, thus explaining why the DNA-binding-defective form of Yan is exported even in the absence of the RTK signal.

In addition to masking the Crm1-binding surface, Yan polymerization is also likely to mask the Ser127 phosphorylation site in Yan, which is immediately adjacent to the SAM domain (Qiao et al. 2004). If, as we suspect, Yan polymerization renders Yan refractory to both Crm1 binding and phosphorylation, how is Yan able to respond to the RTK signal? Recent studies suggest a critical role for Mae, a SAM domain protein that lacks a DNA-binding domain, in this response (Baker et al. 2001; Tootle et al. 2003; Yamada et al. 2003; Qiao et al. 2004; Vivekanand et al. 2004). The binding of Mae SAM to Yan SAM induces Yan depolymerization, leading to the derepression of Yan reporters, but not to Yan nuclear export. The depolymerization of Yan by Mae could expose the Yan phosphorylation site, which would otherwise be buried in the polymer, and thereby poise Yan for response to the RTK signal. In contrast to the results observed for the DNA-binding-defective form of Yan, however, Mae-depolymerized Yan remains in the nucleus in the absence of the RTK signal, perhaps because Mae interferes with binding of Yan to Crm1.

Here we present experiments designed to test the hypothesis that the interaction between Yan and Crm 1 is regulated by both Yan polymerization and the Yan/Mae 
interaction. We show that monomeric Yan is exported from the nucleus in a Crm1-dependent manner. Phosphorylation of Yan facilitates but is not required for this export, while the binding of Mae to Yan prevents the export. Finally, we show that Mae and Crm1 compete for binding to Yan, and that phosphorylation enhances the Crm1-Yan interaction, thereby allowing Crm 1 to prevail in the competition with Mae for binding to Yan. Based on these observations and the finding that the transcription of Mae is negatively regulated by Yan (Vivekanand et al. 2004), we propose a model in which the Mae:Yan interaction serves to regulate Yan nuclear export by both inhibiting export of the Yan monomer in the absence of signaling and facilitating Yan depolymerization and therefore Yan phosphorylation in the presence of signaling. By regulating Yan function at multiple stages in the response to RTK signaling, Mae appears to be critical for the stringent regulation of RTK-response genes in development.

\section{Results and Discussion}

The Yan monomer is exported from the nucleus in a Crm1-dependent manner

Yan polymerization is mediated by two hydrophobic SAM domain interfaces termed the mid-loop (ML) and end-helix (EH) surfaces, which bind one another to form a head-to-tail polymer. Mutagenesis of key residues in these surfaces (e.g., Ala86 on the ML surface or Val105 on the EH surface) converts Yan from a polymer into a monomer (Fig. 1A; Qiao et al. 2004).

In unstimulated Drosophila S2 cells, wild-type Yan remains in the nucleus, while introduction of constitutively active $\operatorname{Ras}\left(\operatorname{Ras}^{\mathrm{V} 12}\right)$ results in Yan export (Fig. 1B,C). In contrast, the two monomeric mutants, Yan ${ }^{\mathrm{A} 86 \mathrm{D}}$ and Yan ${ }^{\mathrm{V} 105 \mathrm{R}}$, are both exported from the nucleus even in the absence of Ras signaling (Fig. 1D,E; data not shown). Nuclear localization was quantified by categorizing cells according to whether they displayed predominant nuclear localization $(\mathrm{N})$, predominant cytoplasmic localization (C), or localization in both the nucleus and cytoplasm $(\mathrm{N}+\mathrm{C})$ (Fig. 1H). Approximately 90\% of cells expressing wild-type Yan displayed predominant nuclear localization. In contrast, only $\sim 20 \%$ of cells expressing monomer Yan displayed predominant nuclear localization, with $\sim 50 \%$ displaying localization in both the nucleus and cytoplasm, and $\sim 30 \%$ displaying predominant cytoplasmic localization. Export of monomeric Yan mutants was fully Crm1-dependent, since cotransfection of dsRNA against Crm1 resulted in predominant nuclear localization of monomeric Yan in $90 \%$ of the cells (Fig.
1F,H). Conversely, overexpression of Crm1 enhanced monomeric Yan export; $>90 \%$ of Yan $^{\mathrm{A} 86 \mathrm{D}}$-expressing cells displayed predominant cytoplasmic localization of monomeric Yan (Fig. 1G,H).

Since unstimulated S2 cells may nonetheless contain low levels of Ras signaling, we determined whether or not monomer export required the critical phosphoacceptor Ser127 residue in Yan. Monomeric Yan harboring the S127A mutation was still exported to the cytoplasm, although to a lesser extent than was monomeric Yan without the S127A mutation. Once again, the export was completely dependent upon Crm1 as demonstrated by Crm1 RNA interference (RNAi) (Fig. 1H). In conclusion, the export of monomeric Yan is dependent on Crm1. Ras signaling stimulates but is not strictly required for export of the monomeric protein.

\section{Mae retains the Yan monomer in the nucleus}

The Mae SAM domain contains a functional ML surface that binds the Yan SAM EH surface, blocking Yan polymerization (Fig. 1A). Mae lacks a functional EH surface and so cannot bind to the Yan ML surface. Because the intrinsic affinity of Yan EH for Mae ML is three orders of magnitude greater than the intrinsic affinity of Yan EH for Yan ML, complete depolymerization of Yan occurs whenever Mae is present in stoichiometric excess over
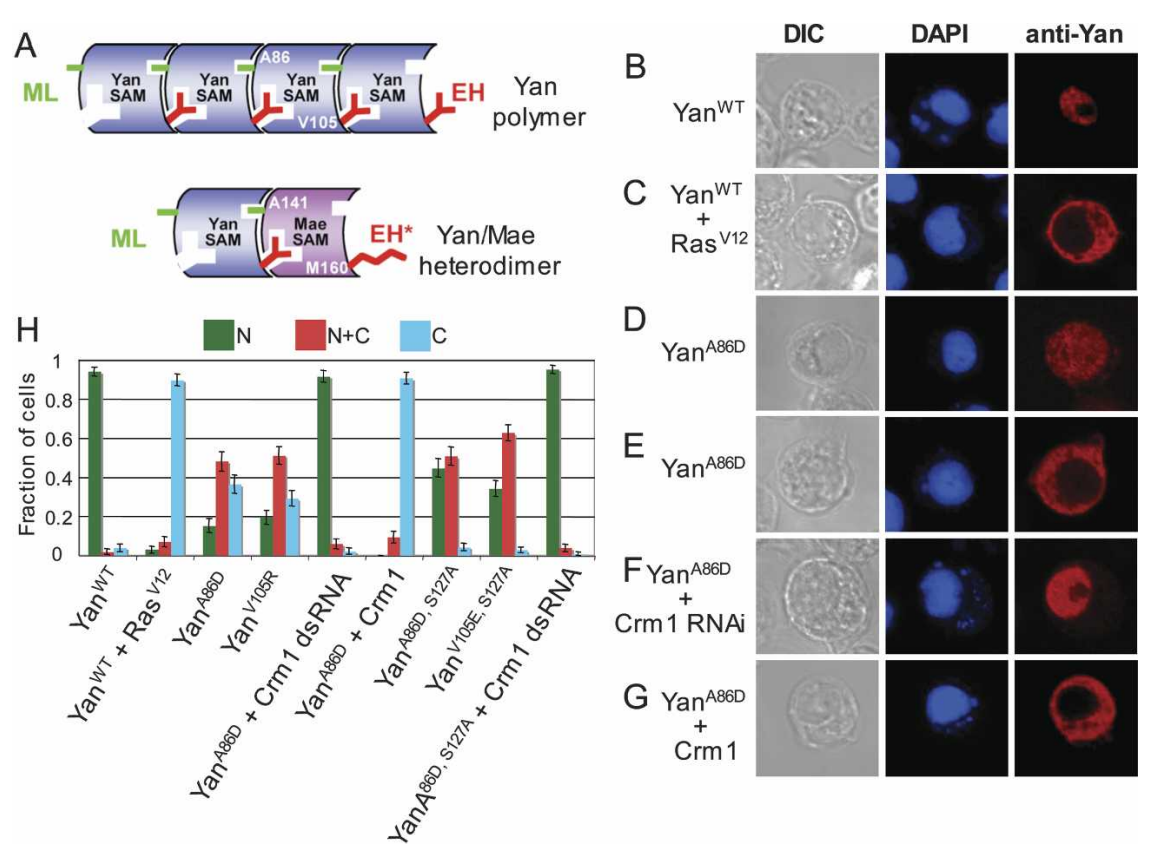

Figure 1. Monomeric Yan is exported from the nucleus in a Crm1-dependent manner. $(A)$ Schematic representation of the Yan SAM polymer and the Yan SAM:Mae SAM heterodimer. The ML surface and the EH surfaces, including key hydrophobic residues required for oligomerization, are indicated. The asterisk $\left(\mathrm{EH}^{\star}\right)$ denotes the naturally nonfunctional EH surface in Mae. $(B-G)$ Cells transfected with constructs encoding the indicated proteins were imaged by differential interference contrast microscopy (DIC; left panels), DAPI staining (which labels the nuclei) and fluorescence microscopy (center panels), or anti-Yan immunofluorescence microscopy (right panels). Wild-type Yan is localized in the nucleus in the absence of Ras signaling $(B)$ and exported to the cytoplasm in the presence of Ras ${ }^{\mathrm{V} 12}$ (activated Ras) (C). The monomeric Yan mutant, Yan ${ }^{\mathrm{A} 86 \mathrm{D}}$, is localized either homogenously throughout the cell $(D)$, or predominantly in the cytoplasm $(E)$. RNAi against Crml results in nuclear localization of $\operatorname{Yan}^{\mathrm{A} 86 \mathrm{D}}(F)$, while overexpression of Crm 1 results in cytoplasmic localization of $\operatorname{Yan}^{\mathrm{A} 86 \mathrm{D}}(G)$. $(H)$ Quantitative analysis of Yan localization. Cells transfected with the indicated constructs were scored as exhibiting predominant nuclear localization $(\mathrm{N})$, predominant cytoplasmic localization $(\mathrm{C})$, or localization in both the nucleus and cytoplasm $(\mathrm{N}+\mathrm{C})$. Error bars indicate \pm standard deviation. 

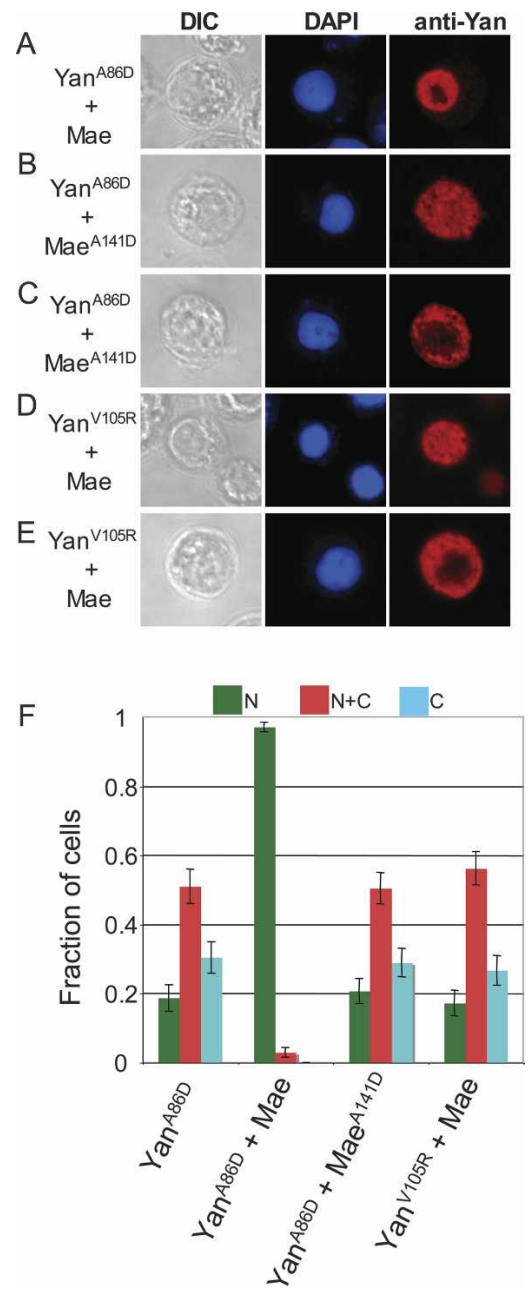

Figure 2. The interaction between Yan and Mae prevents the nuclear export of monomeric Yan. $(A-E)$ Transiently transfected cells were stained and imaged as in Figure 1. (A) Coexpression of wild-type Mae results in the almost complete relocalization of Yan $^{\mathrm{A} 86 \mathrm{D}}$ to the nucleus (cf. Fig. $\left.1 \mathrm{D}, \mathrm{E}\right) .(B, C)$ However, Mae ${ }^{\mathrm{A141D}}$ does not interfere with Yan ${ }^{\mathrm{A} 86 \mathrm{D}}$ localization, and thus the cells display either homogeneous localization $(B)$ or cytoplasmic localization $(C)$ (cf. Fig. 1D,E). $(D, E)$ Wild-type Mae does not interfere with the nuclear export of $\mathrm{Yan}^{\mathrm{V} 105 \mathrm{R}}$, and thus the cells display either homogeneous localization $(D)$ or cytoplasmic localization $(E)$. (F) Quantitative analysis of Yan localization was as described in Figure $1 \mathrm{H}$.

Yan. Under these conditions, all the Yan is driven into Yan:Mae heterodimers (Qiao et al. 2004).

If nuclear export of the Yan monomer is due to the exposure of Crm1-binding site(s) in the monomer that are buried in the Yan polymer, the Yan:Mae interaction might also mask the Crm1-binding site(s), resulting in the nuclear retention of the monomer. In accord with this idea, cotransfection of Mae prevented the nuclear export of $\mathrm{Yan}^{\mathrm{A} 86 \mathrm{D}}$, a monomeric Yan mutant that retains the ability to bind Mae (Fig. 2A,F). The nuclear retention of the Yan monomer was completely dependent upon the interaction between Yan and Mae, since a Mae mutant with a defective ML surface, which is unable to bind to Yan, fails to interfere with the export of Yan ${ }^{A 86 D}$ (Fig. 2B,C,F). In addition, the localization of Yan ${ }^{\mathrm{V} 105 \mathrm{R}}$, a monomeric Yan mutant that cannot bind
Mae, was not altered by cotransfection of wild-type Mae (Fig. 2D-F).

The Mae-mediated nuclear retention of Yan is antagonized by Crm1 and Ras signaling

If Mae masks the Crm1-binding site(s) on Yan, then Crm1 might compete with Mae for binding to Yan. In this case, Crm 1 overexpression would be expected to antagonize nuclear retention of Yan by Mae. In addition, if Yan phosphorylation facilitates Yan export by helping Crm1 to compete with Mae, cotransfection of Ras ${ }^{12}$ should also antagonize Mae-mediated Yan nuclear retention. To test these possibilities we coexpressed Yan ${ }^{\mathrm{A} 86 \mathrm{D}}$ and Mae in the presence of Ras ${ }^{\mathrm{V} 12}$, Crm1, or both (Fig. 3). Overexpression of Mae resulted in predominant nuclear localization of monomeric Yan in $\sim 85 \%-95 \%$ of the cells. Coexpression of Crm 1 reduced this proportion to $\sim 20 \%-30 \%$, dependent on the dose of transfected Mae. Coexpression of Ras ${ }^{\mathrm{V} 12}$ alone had a much smaller effect on Mae-mediated Yan nuclear retention-the proportion of cells with predominant nuclear localization was $\sim 65 \%-85 \%$. The level of Ras ${ }^{\mathrm{V} 12}$ expression used in these experiments is sufficient to drive essentially all Yan from the nucleus in the absence of cotransfected Mae (see Fig. 1C). Coexpression of Ras ${ }^{\mathrm{V} 12}$ and Crm1 resulted in additive or slightly greater than additive effects on monomeric Yan nuclear retention, supporting the idea that Ras signaling facilitates Crm 1-mediated Yan export.

\section{Crm1 and Mae compete for binding to Yan}

To test directly for competition between Crm1 and Mae, we employed coprecipitation assays. As the interaction between Crm 1 and its targets is affected by multiple factors (e.g., it is stabilized by RanGTP in the nucleus and destabilized by RanGDP in the cytoplasm, and may depend upon additional adaptors; Fornerod et al. 1997; Nakielny and Dreyfuss 1999; Englmeier et al. 2001; Kuersten et al. 2001; Petosa et al. 2004), we employed S2 cell nuclear extracts as a source of Crm1. Transiently expressed Flag-tagged Yan was purified on anti-Flag agarose beads, and then incubated with S2 nuclear extract. Crm1 precipitated with the Flag-Yan-bound beads (Fig. 4A, lane 2), but not with the beads alone (Fig. 4A, lane 1). The

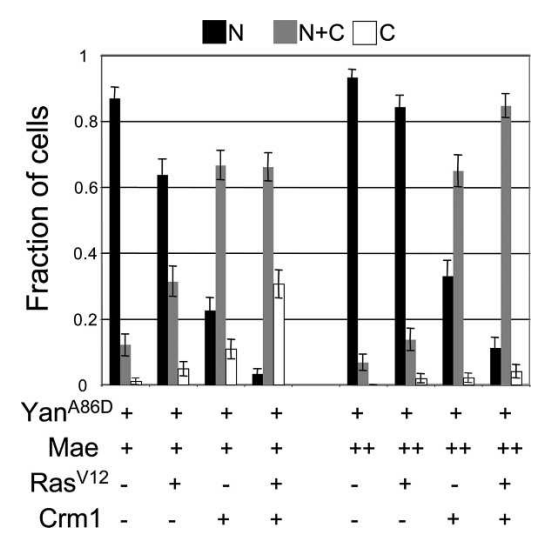

Figure 3. Nuclear localization of the Yan monomer by Mae is antagonized by Crm 1 and Ras signaling. The monomeric Yan mutant, Yan ${ }^{\mathrm{A} 86 \mathrm{D}}$, was expressed in the presence of the indicated combinations of $\mathrm{Mae}, \mathrm{Crm} 1$, and Ras ${ }^{\mathrm{v} 2}$. The experiments were carried out at low $(+)$ or high $(++)$ concentrations of Mae. The data were quantified as described in the legend for Figure 1H. 
Song et al.

A

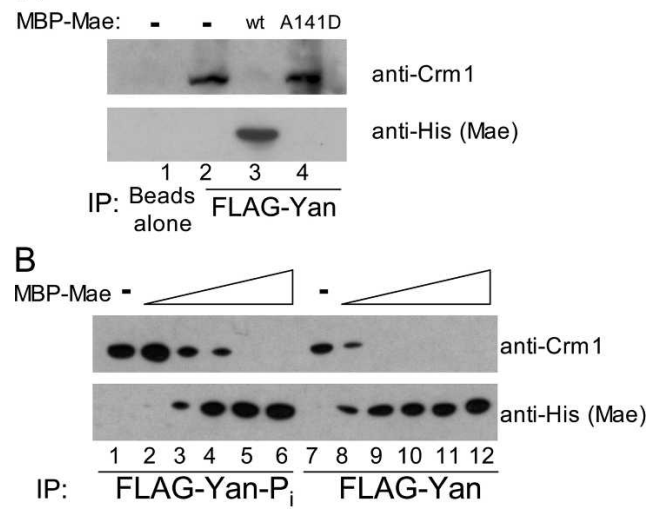

C

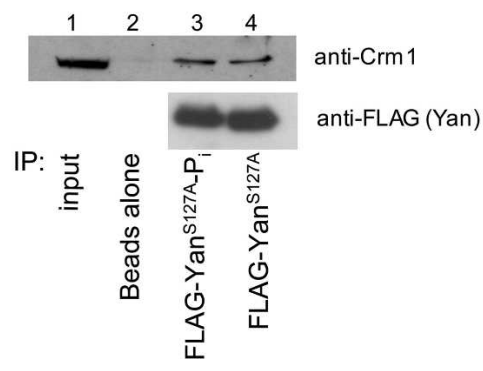

Figure 4. Antagonism between $\mathrm{Crm} 1$ and Mae for binding to Yan is modulated by Yan phosphorylation. (A) Crml and Mae compete for Yan binding. Flag-tagged Yan purified from S2 cells was immobilized on anti-Flag agarose beads, and S2 cell nuclear extracts were incubated with the beads in the absence (lane 2) or presence of bacterially expressed His-tagged MBP-MaeSAM (lane 3) or MBP-

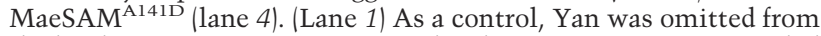
the binding reaction. Coprecipitated endogenous $\mathrm{Crm} 1$ was revealed by anti-Crm1 immunoblotting (upper panel), while coprecipitated Mae was revealed by anti-His immunoblotting (lower panel). (B) Immobilized Flag-Yan that had been expressed in S2 cells in the presence (lanes 1-6) or absence (lanes 7-12) of Ras ${ }^{\mathrm{V} 12}$ was incubated with Crm1-containing nuclear extract and variable amounts of bacterially expressed MBP-MaeSAM. Coprecipitated endogenous Crm1 was revealed by anti-Crml immunoblotting (upper panel), while coprecipitated Mae was revealed by anti-His immunoblotting (lower panel). (C) Immobilized Flag-Yan ${ }^{\text {S127A }}$ that had been expressed in S2 cells in the presence (lane 3) or absence (lane 4) of Ras ${ }^{\mathrm{V} 12}$ was incubated with Crm1-containing nuclear extract. Also shown are $15 \%$ of the input Crml (lane 1) and a negative control in which Yan was omitted from the binding reaction (lane 2). Coprecipitated endogenous Crm1 was revealed by anti-Crm1 immunoblotting (upper panel), while precipitated recombinant Yan was revealed by antiFlag immunoblotting (lower panel).

bound Crm 1 was displaced from the beads upon addition of the wild-type MBP-MaeSAM domain (Fig. 4A, lane 3), but not upon addition of MBP-MaeSAM ${ }^{\mathrm{A} 141 \mathrm{D}}$, in which the SAM domain contains a point mutation that disrupts the ML surface (Fig. 4A, lane 4).

We next determined whether phosphorylation of Yan enhanced the coprecipitation of Crm1. Flag-tagged wildtype Yan was transiently expressed in S2 cells in the absence or presence of Ras ${ }^{\mathrm{V} 12}$ and then tested in coprecipitation assays for binding to Crm 1 in S2 cell nuclear extracts. Like untagged Yan, Flag-tagged Yan localizes to the nucleus in the absence of Ras signaling and is exported to the cytoplasm in the presence of Ras signaling (data not shown). The amount of Ras ${ }^{\mathrm{V} 12}$ used in these experiments was sufficient to drive nearly all the Yan protein into the cytoplasm, indicating that Yan phos- phorylation is likely quantitative. This was further verified by anti-Flag immunoblots showing a quantitative mobility shift of Flag-Yan upon coexpression with Ras $^{\mathrm{V} 12}$ (data not shown). The Crm1-Yan interaction was more stable when the Yan was expressed with the activated form of Ras, as shown by the coprecipitation and Mae competition assays (Fig. 4B, cf. lanes 1-6 and 7-12). This enhanced interaction was likely dependent on phosphorylation of Yan on Ser127, as coexpression with activated Ras had no effect on the ability of Flag-tagged Yan $^{\text {S127A }}$ to bind Crm1 (Fig. 4C, cf. lanes 3 and 4).

In contrast to the results described above in which we employed a Crm1-containing nuclear extract, purified Crm1 does not show significant affinity for Yan (data not shown), indicating that the nuclear extract provides additional components that stabilize the interaction. This is consistent with previous studies on exportins showing that they often require adaptor molecules for high-affinity binding to their targets (Englmeier et al. 2001; Kuersten et al. 2001). In addition, the GTP-bound form of Ran is required for the stable binding of Crm1 to its targets (Fornerod et al. 1997; Nakielny and Dreyfuss 1999; Petosa et al. 2004), and we therefore included GMP-PNP as a standard component in all the Crm1 binding assays.

$A$ model for the regulation of the Crm1:Yan interaction

We propose a model in which Mae plays multiple roles in regulating the Crm1:Yan interaction. In cells that

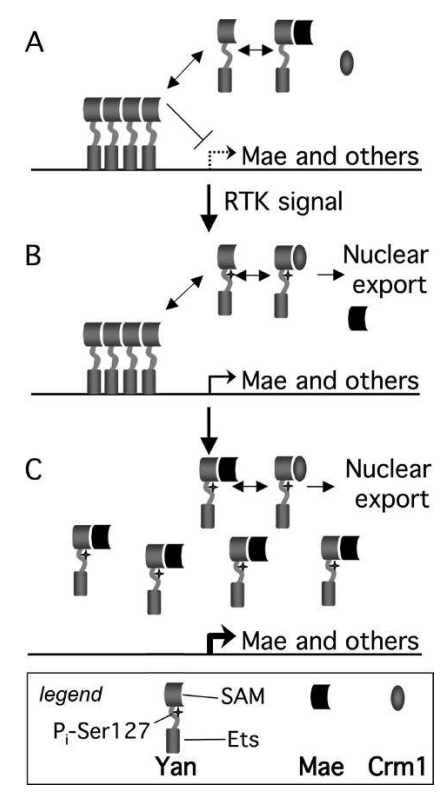

Figure 5. Proposed model for the regulation of Yan localization by antagonism between Crm1 and Mae. (A) In unstimulated cells, polymeric and monomeric Yan are in equilibrium with one another. Favorable binding of the small amount of Mae present in these cells to monomeric Yan pulls the equilibrium away from the polymer. Since depolymerization is required for the access of MAPK to the Yan phosphorylation site, this helps to poise Yan for response to the RTK signal. At the same time, Mae prevents access of Crm1 to monomeric Yan. (B) Upon RTK activation, phosphorylation of the Yan monomer favors Crm 1 binding, leading to the export of the Yan monomer. Increased export of Yan results in the partial derepression of Yan target genes including Mae. $(C)$ Increased levels of Mae completely depolymerize Yan, leading to full derepression of Yan targets. 
have not received the RTK signal, polymeric Yan is in equilibrium with depolymerized Yan (Fig. 5A). The formation of the depolymerized Yan is likely favored by the presence of a small amount of Mae in the nucleus of unstimulated cells, which binds and blocks one of the Yan polymerization interfaces (the Yan EH surface). We suspect that by promoting limited Yan depolymerization, Mae ensures that unstimulated cells will be poised to receive the RTK signal. However, at this stage Mae also appears to block access of Crm1 to depolymerized Yan. This is important since if depolymerized Yan was accessible to Crm1, the resulting export of Yan would pull the equilibrium away from the polymer, leading to further Yan export and ultimately to premature expression of Yan target genes.

It is essential to maintain Mae at low levels in undifferentiated cells, because, as we have shown, Mae-mediated Yan depolymerization is sufficient to result in derepression, even in the absence of Ras signaling (Qiao et al. 2004). Maintenance of low levels of Mae prior to differentiation appears to be at least partially ensured by the Yan-dependent repression of Mae (Vivekanand et al. 2004). Upon activation of Ras, Ser127 in the Yan-Mae dimer is phosphorylated by the activated Rolled MAPK, favoring Crm1 in the competition with Mae for binding to Yan (Fig. 5B). The resulting increased Yan export is expected to relieve the transcriptional repression of Mae, and the accumulation of additional Mae is then expected to result in further Yan depolymerization, phosphorylation, and nuclear export (Fig. 5C). This feedback loop ensures that while Yan targets will be stably repressed prior to differentiation, they will be completely derepressed upon reception of the RTK signal. Furthermore, this feedback loop could serve to amplify a small change in the level of the RTK ligand, thereby allowing for sharp threshold responses to graded extracellular ligands.

\section{Materials and methods}

\section{Plasmids}

Yan $^{\mathrm{A} 86 \mathrm{D}, \text { S127A }}$ and Yan ${ }^{\mathrm{V} 105 \mathrm{E}, \text { S127A }}$ expression plasmids were constructed by site-directed mutagenesis of $\mathrm{pRM}-\mathrm{Yan}^{\mathrm{S} 127 \mathrm{~A}}$ (kindly provided by Ilaria Rebay, University of Chicago, Chicago, IL). Expression plasmids encoding the N-terminally Flag-tagged Yan proteins were constructed by inserting PCR-derived fragments between the KpnI and SacI sites of the pPac-Flag vector (Chen et al. 1999). The Crml coding region was PCRamplified from a pGEX-Crml plasmid (kindly provided by Christos Samakovlis, Stockholm University, Stockholm, Sweden) and inserted into a pAct vector (Invitrogen). Other plasmids have been previously described (Qiao et al. 2004).

RNAi

The primers for making Crm1 dsRNA were designed as previously described (Tootle et al. 2003). Cells were collected 3 d after transfection.

\section{Immunohistochemistry}

S2 cells were transfected by the calcium phosphate precipitation procedure. Immunofluorescent staining was carried out as previously described (Qiao et al. 2004). Rabbit polyclonal anti-Crml antibody was kindly provided by Christos Samakovlis. Mouse monoclonal anti-Flag antibody and anti-Flag agarose beads were from Sigma. Mouse monoclonal anti-His HRP-conjugated antibody was from Invitrogen.

\section{Coprecipitation assays}

S2 cells transfected with Flag-tagged Yan were lysed in buffer A (50 mM Tris- $\mathrm{HCl}$ at $\mathrm{pH} 7.4,150 \mathrm{mM} \mathrm{NaCl}, 2 \mathrm{mM}$ EDTA, 2 mM EGTA, 0.8\% Triton X-100, $0.5 \mathrm{mM}$ DTT and protease inhibitor cocktail; Roche). Clarified lysates were incubated with anti-Flag agarose beads, washed with buffer $\mathrm{A}$ and then with pull-down buffer $(50 \mathrm{mM}$ Tris- $\mathrm{HCl}$ at $\mathrm{pH} 7.4$, $150 \mathrm{mM} \mathrm{NaCl}, 1 \mathrm{mM}$ DTT). MBP-MaeSAM was purified as previously described (Qiao et al. 2004). To prepare S2 cell nuclear extracts, cell membranes were broken in buffer B $(10 \mathrm{mM}$ Tris- $\mathrm{HCl}$ at $\mathrm{pH} 7.4,10 \mathrm{mM}$ $\mathrm{NaCl}, 50 \mathrm{mM} \mathrm{KCl}, 2 \mathrm{mM} \mathrm{MgCl}_{2}, 0.75 \% \mathrm{NP} 40,0.5 \mathrm{mM} \mathrm{DTT}$, and protease inhibitor cocktail; Roche). The nuclei were pelleted, resuspended in buffer C (50 mM Tris-HCl at pH 7.4, 250 mM NaCl, 2 mM EDTA, 2 mM EGTA, 1 mM GMP-PNP [Sigma], 0.5\% Triton X-100, 10\% glycerol, 0.5 mM DTT, and protease inhibitor cocktail [Roche]) and incubated at $4^{\circ} \mathrm{C}$ for $1 \mathrm{~h}$. Two-hundred microliters of clarified nuclear extract was diluted with $800 \mu \mathrm{L}$ pull-down buffer containing 1 mM GMP-PNP and incubated with the Flag-Yan beads. After extensive washing with pull-down buffer, the bound protein was eluted with SDS-PAGE sample buffer and analyzed by Western blotting.

\section{Acknowledgments}

We thank Songtao Jia for helpful discussions, Ilaria Rebay for Yan and Mae constructs, and Christos Samakovlis for Crm 1 constructs and Crm1 antibody. This work was supported by grants RO1 GM44522 and RO1 GM63596 to A.J.C. and by NIH grant R01 CA081000 to J.U.B. J.U.B. is a Leukemia and Lymphoma Society Scholar.

\section{References}

Baker, D.A., Mille-Baker, B., Wainwright, S.M., Ish-Horowicz, D., and Dibb, N.J. 2001. Mae mediates MAP kinase phosphorylation of Ets transcription factors in Drosophila. Nature 411: 330-334.

Chen, G., Fernandez, J., Mische, S., and Courey, A.J. 1999. A functional interaction between the histone deacetylase Rpd3 and the corepressor groucho in Drosophila development. Genes \& Dev. 13: 22182230.

Englmeier, L., Fornerod, M., Bischoff, F.R., Petosa, C., Mattaj, I.W., and Kutay, U. 2001. RanBP3 influences interactions between CRM1 and its nuclear protein export substrates. EMBO Rep. 2: 926-932.

Fornerod, M., Ohno, M., Yoshida, M., and Mattaj, I.W. 1997. CRM1 is an export receptor for leucine-rich nuclear export signals. Cell 90: 10511060.

Freeman, M. 1996. Reiterative use of the EGF receptor triggers differentiation of all cell types in the Drosophila eye. Cell 87: 651-660.

Gabay, L., Scholz, H., Golembo, M., Klaes, A., Shilo, B.Z., and Klambt, C. 1996. EGF receptor signaling induces pointed P1 transcription and inactivates Yan protein in the Drosophila embryonic ventral ectoderm. Development 122: 3355-3362.

Klambt, C. 1993. The Drosophila gene pointed encodes two ETS-like proteins which are involved in the development of the midline glial cells. Development 117: 163-176.

Kuersten, S., Ohno, M., and Mattaj, I.W. 2001. Nucleocytoplasmic transport: Ran, $\beta$ and beyond. Trends Cell Biol. 11: 497-503.

Lai, Z.C. and Rubin, G.M. 1992. Negative control of photoreceptor development in Drosophila by the product of the yan gene, an ETS domain protein. Cell 70: 609-620.

Nakielny, S. and Dreyfuss, G. 1999. Transport of proteins and RNAs in and out of the nucleus. Cell 99: 677-690.

O'Neill, E.M., Rebay, I., Tjian, R., and Rubin, G.M. 1994. The activities of two Ets-related transcription factors required for Drosophila eye development are modulated by the Ras/MAPK pathway. Cell 78: $137-147$.

Petosa, C., Schoehn, G., Askjaer, P., Bauer, U., Moulin, M., Steuerwald, U., Soler-Lopez, M., Baudin, F., Mattaj, I.W., and Muller, C.W. 2004. Architecture of CRM1/Exportin1 suggests how cooperativity is achieved during formation of a nuclear export complex. Mol. Cell 16: 761-775

Qiao, F., Song, H., Kim, C.A., Sawaya, M.R., Hunter, J.B., Gingery, M., Rebay, I., Courey, A.J., and Bowie, J.U. 2004. Derepression by depolymerization; structural insights into the regulation of Yan by Mae. Cell 118: 163-173.

Rebay, I. and Rubin, G.M. 1995. Yan functions as a general inhibitor of differentiation and is negatively regulated by activation of the Ras1/ MAPK pathway. Cell 81: 857-866.

Shilo, B.Z. 2003. Signaling by the Drosophila epidermal growth factor receptor pathway during development. Exp Cell Res. 284: 140-149. 
Song et al.

Shvartsman, S.Y., Muratov, C.B., and Lauffenburger, D.A. 2002. Modeling and computational analysis of EGF receptor-mediated cell communication in Drosophila oogenesis. Development 129: 2577-2589.

Tan, P.B. and Kim, S.K. 1999. Signaling specificity: The RTK/RAS/MAP kinase pathway in metazoans. Trends Genet. 15: 145-149.

Tootle, T.L., Lee, P.S., and Rebay, I. 2003. CRM1-mediated nuclear export and regulated activity of the receptor tyrosine kinase antagonist YAN require specific interactions with MAE. Development 130: 845857.

Vivekanand, P., Tootle, T.L., and Rebay, I. 2004. MAE, a dual regulator of the EGFR signaling pathway, is a target of the Ets transcription factors PNT and YAN. Mech. Dev. 121: 1469-1479.

Wassarman, D.A., Therrien, M., and Rubin, G.M. 1995. The Ras signaling pathway in Drosophila. Curr. Opin. Genet. Dev. 5: 44-50.

Yamada, T., Okabe, M., and Hiromi, Y. 2003. EDL/MAE regulates EGFmediated induction by antagonizing Ets transcription factor Pointed. Development 130: 4085-4096. 


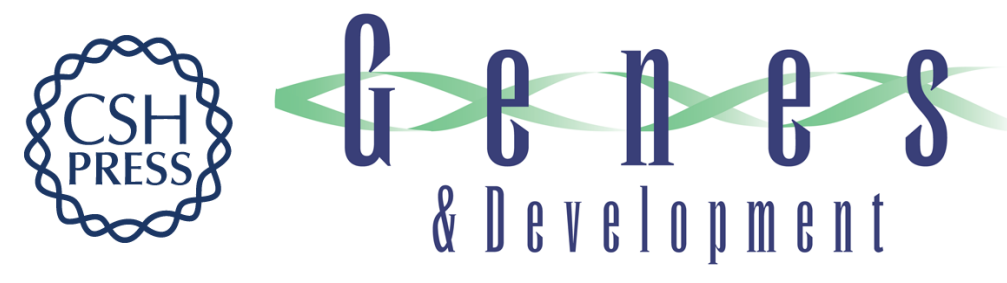

\section{Antagonistic regulation of Yan nuclear export by Mae and Crm1 may increase the stringency of the Ras response}

Haiyun Song, Minghua Nie, Feng Qiao, et al.

Genes Dev. 2005, 19:

Access the most recent version at doi:10.1101/gad.1327405

References

This article cites 21 articles, 7 of which can be accessed free at:

http://genesdev.cshlp.org/content/19/15/1767.full.html\#ref-list-1

\section{License}

Email Alerting

Receive free email alerts when new articles cite this article - sign up in the box at the top

Service right corner of the article or click here.

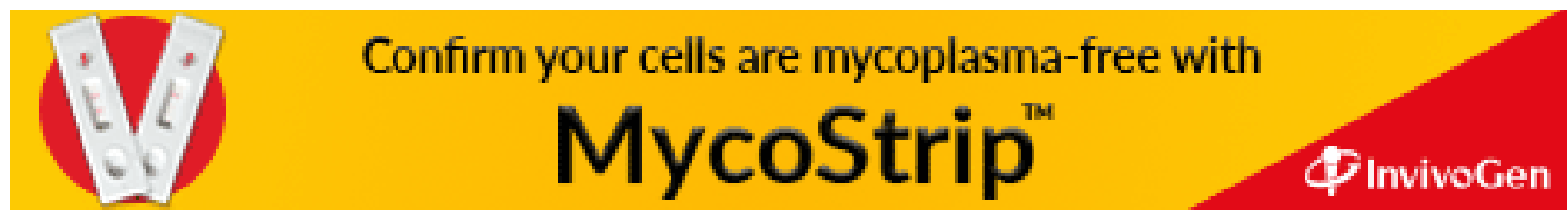

